\title{
Specimen Processing
}

National Cancer Institute

\section{Source}

National Cancer Institute. Specimen Processing. NCI Thesaurus. Code C41604.

The process of preparing a sample or portion of a sample for storage, preservation, or further analysis. 